Journal of Reproduction and Development, Vol. 39, No. 3, 1993

\title{
Ultrastructural Evidence for Crossing Over in the XY Bivalent of the Turkish Hamster (Mesocricetus brandti)
}

\author{
Weng Kong SUNG*, Georgiana M. JAGIELLO') and \\ Tadahiro INOUE
}

National Institute of Animal Health, Kannondai, Tsukuba, Ibaraki 305, Japan, and

${ }^{1)}$ Departments of Obstetrics and Gynecology, Genetics and Development and the Center for Reproductive Sciences, Columbia University, College of Physicians and Surgeons, 630 West 168th Street, New York, N.Y. 10032, USA

\begin{abstract}
A sequential electron microscopic study of XY synaptonemal complexes of male Turkish hamster sprematocytes by 2 methods was carried out. The results revealed that 1 or 2 chromatin fibril-like strands were commonly extending and connecting the $\mathrm{XY}$ axes either around the border of the differential and non-differential segments or near the attachment plaques. The existence of such strands between 2 axes, and the number and position they presented were found to be highly correlating to stages/sub-stages of the spermatocytes. A possibility of visual evidence for chiasma formation and terminalization in the sex bivalent in the present species is discussed.
\end{abstract}

Key words: Turkish hamster, XY pair, Chiasma, Chromosome, Meiotic stage.

(J. Reprod. Dev. 39: 207-216, 1993)

$\mathbf{T}$ he existence of crossing over between the sex chromosomes in male mammalian meiosis has been debated since it was first proposed by Koller and Dalington [1]. Early morphologic observations which revealed the $\mathrm{X}$ and $\mathrm{Y}$ chromosomes in an end-to-end association without a visible chiasma contributed to the debate. Recently an "X-Y crossing over" model was postulated by Burgoyne $[2,3]$. This was based on a proposed etiology of Turner's syndrome [4] and the transmission of a Y-linked DNA probe in XY Sxr male mouse [5] which indicated that the pairing of the $\mathrm{XY}$ chromosomes was a consequence of genetic homology with a single obligatory crossover between the $\mathrm{X}$ and $\mathrm{Y}$ pairing segments. This proposition has been supported by several genetic and molecu-

Accepted for Publication: April, 14, 1993

Correspondence: $T$. Inoue

* Research Scientist of STA (The Science and Technology

Agency Japan) Fellowship. lar observations [6-14] and has been accepted as a general rule for the occurrence of crossing over between the XY chromosomes in mammals [15-17] despite a few exceptions [18-24]. Thus, it is currently believed that a genetic exchange occurs between the $\mathrm{X}$ and $\mathrm{Y}$ by a single $[2,7,10,11]$ or double [13] obligatory crossing over taking place at the so-called "pseudoautosomal region" in the tip of the two sex chromosomes. In the present study, a possible morphological evidence was provided for the formation of the chiasma between the XY chromosomes in the Turkish hamster that may support both early [1] and recent [2,3] postulates on the existence of $\mathrm{XY}$ crossing over.

\section{Materials and Methods}

Testicular specimens were obtained from 12 adult male Turkish hamsters (Mesocricetus brandti) 
aged 10-15 months. The animals were sacrificed with chloroform and testes were rapidly removed. The first method used for preparing spread synaptonemal complexes was based on Trés and Kierszenbaum [25] with a modification by Sung and Jagiello [26]. Nuclei were spread as whole mounts and the grids were stained with $1 \%$ PTA. A second spreading technique described by Moses [27] combined with silver nitrate staining [28] was also employed to test the findings obtained by the first method. A total of 4 grids, two from each method, were utilized from each donor testis for electron microscopic analyses with a JEOL 100 CX. All spread nuclei with a clearly visible and identifiable XY pair were photographed at low magnification (X600-1300) and higher magnification (X5000-10000). The criteria applied for staging the spermatocytes were those of Trés and Kierszenbaum [25] based in the autosomal characteristics of synaptonemal complexes as applied to other mammals, and for substaging pachytene cells according to the criteria employed for deer mouse spermatocytes by Greenbaum et al. [29].

Additional observations were made of the "endto-end" XY association at the diakinesis stage in cytogenetic preparation stained with silver-nitrate [30]. Briefly, a sample of testis was dissected into several segments in $0.05 \mathrm{M} \mathrm{KCl}$ and kept at the room temperature for $1 \mathrm{~h}$. After fixation with methanol-glacial acetic acid (3:1), the testicular segments were smeared on clean slides, dried and stained with $70 \%$ silver nirate in a dark, wet chamber at $60 \mathrm{C}$ for $48 \mathrm{~h}$.

\section{Results}

As in most mammals, the sex bivalent in this species became distinguishable from the autosomes and initiated synapsis in a small region near the attachment plaques in the late zygotene stage (Fig. 1). Nuclei with pre-synaptic XY axes or with incomplete autosomes technically unsuitable for determining stages/substages by the criteria used were excluded from the analyses. A total of 227 nuclei, 112 from the first spreading method stained with PTA and 115 from the second method stained with silver nitrate were analyzed. The conjugation of the XY axes showed either side-by-side pairing or end-to-end association. As Solari [31] reported in human spermatocytes, various degrees of excrescences and splittings were also found in the $\mathrm{XY}$ axes of the present species throughout the late zygotene to the diplotene stages. The excrescences seemed to be more conspicuous and the splittings became more distinct after the mid-pachytene stage. Both excrescences and splittings were more evident in the differential segment than the paired segments. Commonly, the splitting was so conspicuous that a doubleness or a multistrandes-like structure was evident along the unpaired segments. However, in more than $60 \%$ of analyzed nuclei 1 or 2 fine chromatin fibril-like strands extending and connecting the $\mathrm{XY}$ axes either around the border of the differential and non-differential segments or near the attachment plaques were seen. Based on whether or not such strands existed between the two axes, and the number and position they presented, all XY pairs could be classified into 6 types as follows:

Type 1: The $\mathrm{X}$ and $\mathrm{Y}$ chromosomes paired side-by-side without any connecting strand between the 2 axes (Fig. 2).

Type 2: The XY bivalent paired as with type 1 except a fine strand appeared in the area between paired and unpaired segments from either $\mathrm{X}$ or $\mathrm{Y}$ to the other thus connecting the 2 axes (Fig. 3).

Type 3: The $\mathrm{X}$ chromosome had double structures extending from both sides of the attachment plaques and the $\mathrm{Y}$ chromosome was connected to one of the strands by 1 or 2 strands (Fig. 4).

Type 4: The side-by-side conformation remained unchanged but the portion involved in pairing was decreased. Both axes possessed double stands and each of the inner strands extended to the other forming an X-shaped cross in the space interspersed between the 2 outer strands (Fig. 5).

Type 5: The XY axes paired in the same manner as Type 4 except there was a single strand from one axis to the other; usually from the $\mathrm{Y}$ to the $\mathrm{X}$ chromosome (Fig. 6).

Type 6: The X-Y pairing showed end-to-end association with several fine fibrillar threads connected to 2 attachment plaques (Fig. 7). In some cases the threads 


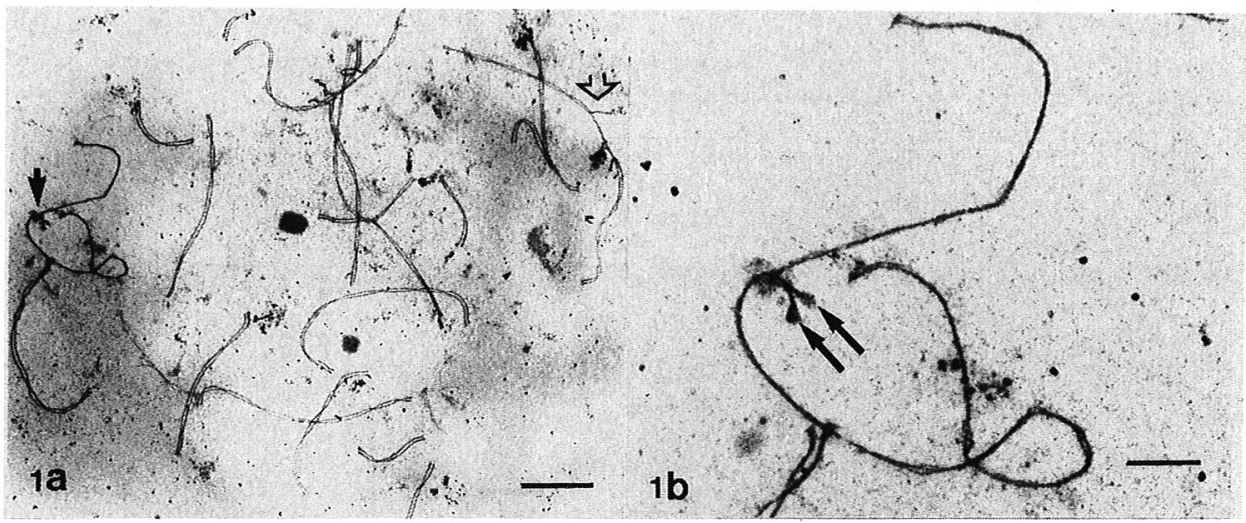

Fig. 1. Electron micrographs of synaptonemal complexes of Turkish hamster spermatocytes. (Silver Stained) a) Overview of nuclei showing late zygotene stage: autosomal pairing almost completed. Scale represents $10 \mu \mathrm{m}$. b) Initiation of XY pairing at a small region near attachment plaques of 2 axes (arrows). Scale represents $50 \mu \mathrm{m}$.

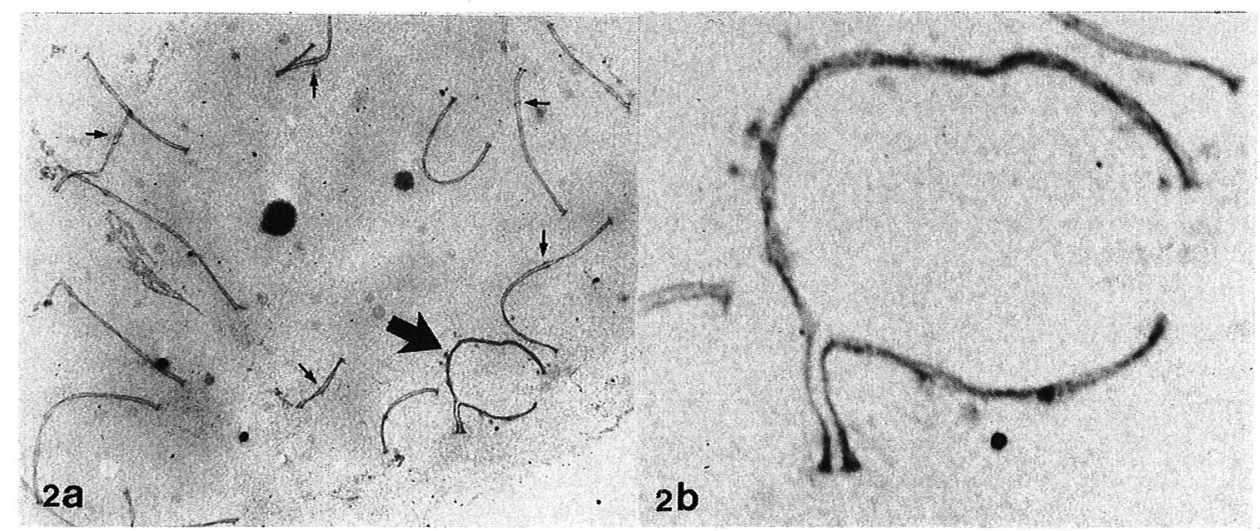

Fig. 2. Electron micrographs of synaptonemal complexes of Turkish hamster spermatocytes. (PTA-stained.) a) Overview of nuclei showing early pachytene stage (P1-2): autosomes show complete synapsis. Axes are still elongated, less condensed and have twists. Large arrow indicates the XY pair and small arrows indicates the kinetochores of autosomes. Magnification is the same as in Fig. 1. b) Enlargements of XY pair showing type 1 configuration; XY showing side-by-side association. Without any connection between the 2 axes. Magnification is the same as in Fig. 1.

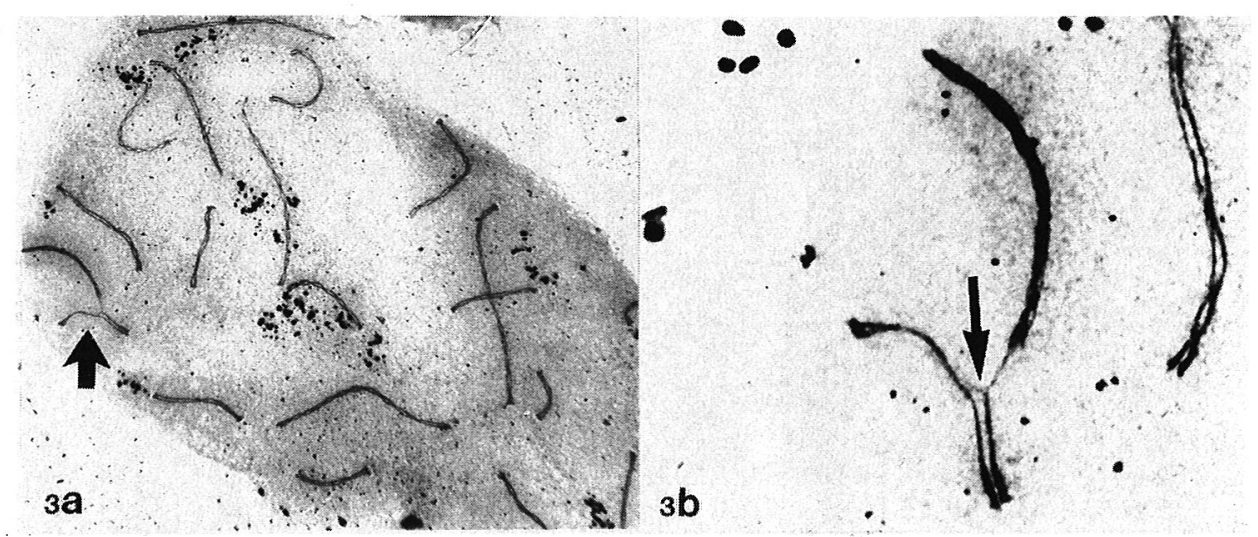

Fig. 3. Electron micrographs of synaptonemal complexes of Turkish hamster spermatocytes. (Silver Stained) a) Overview of nuclei showing early to mid pachytene stage (P2+P3); autosomal axes are increasingly twisted (small arrows). Large arrow indicates XY pair. Magnification is the same as in Fig. 1. b) Enlargements of XY pair showing type 2 configuration; XY bivalent paired as with Type 1 except a fine strand (arrow) connects the 2 axes. Magnification is in the same as Fig. 1. 


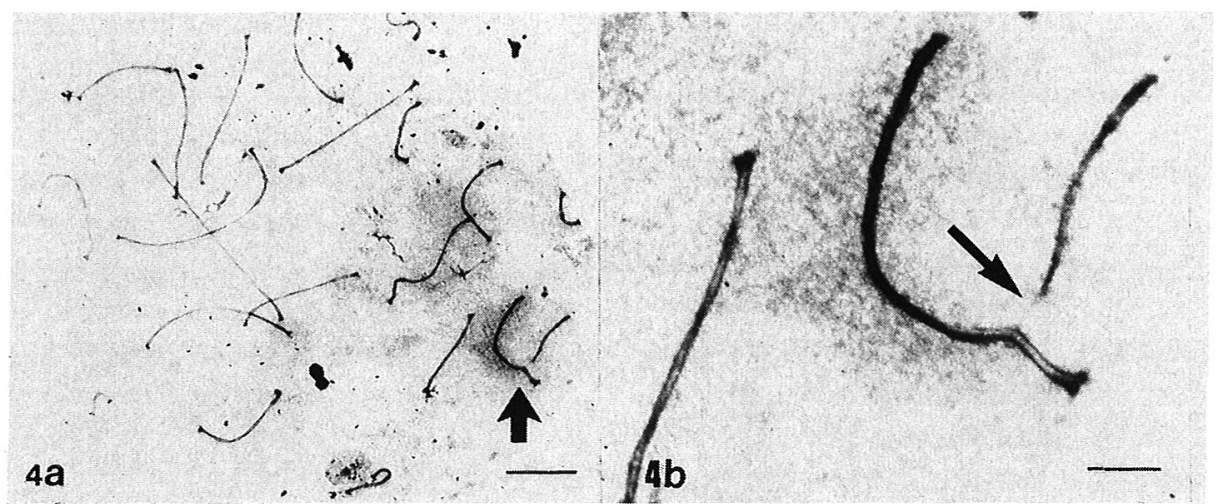

Fig. 4. Electron micrographs of synaptonemal complexes of Turkish hamster spermatocytes. (PTA-stained.) a) Overview of nuclei showing mid pachytene stage (P3); autosomal bivalents are condensing with length reduced. Arrow indicates XY pair. Scale represents $10 \mu \mathrm{m}$. b) Enlargements of XY pair type 3 configuration; $\mathrm{X}$ axis shows a double structure extending from both sides of attachment plaques and $\mathrm{Y}$ axis connects to the inner strand of the $\mathrm{X}$ with a fine strand (arrow). Scale represents $50 \mu \mathrm{m}$.

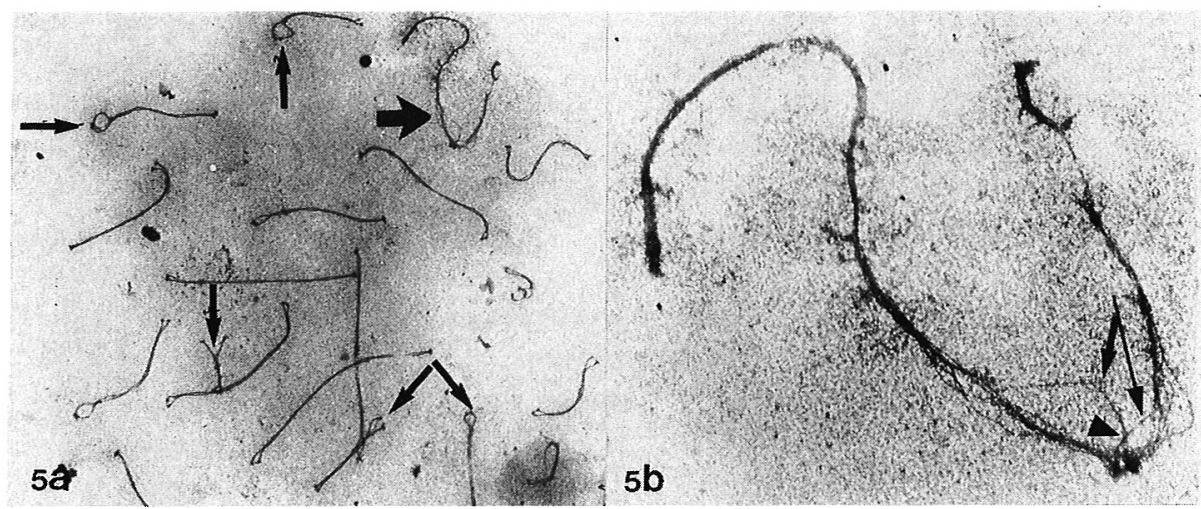

Fig. 5. Electron micrographs of synaptonemal complexes of Turkish hamster spermatocytes. (Silver Stained.) a) Overview of nuclei showing late pachytene stage (P4-P5); autosomal bivalents are thinner and desynapsis is initiating at the end of the axes (small arrows). Large arrow indicates XY pair. Magnification is the same as in Fig. 4. b) Enlargements of XY pair showing type 4 configuration; portion involved in pairing is decreased. Both axes have multistrands. One of the inner strands from each attachment plaque extends to the other (arrows) and crisscrosses the other (arrow head) types of configurations. Magnification is the same as in Fig. 4.

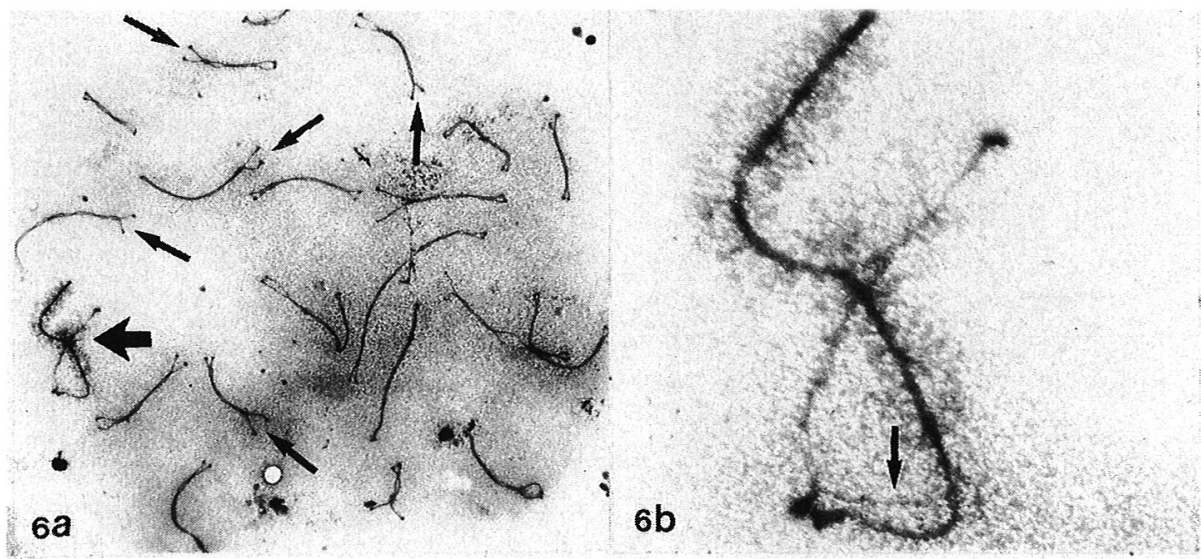

Fig. 6. Electron micrographs of synaptonemal complexes of Turkish hamster spermatocytes. (Silver Stained.) a) Overview of nuclei showing Late pachytene (P5) to early diplotene stage. Onset of desynapsis in autosomes is more clearly evident (small arrows); The XY pair is indicated by large arrows. Magnification is the same as in Fig. 4. b) Enlarged view of XY pair showing type 5 configuration; compared to Type 4, only one inner strand extends from $\mathrm{X}$ to $\mathrm{Y}$ axis (arrows). Magnification is the same as in Fig. 4. 


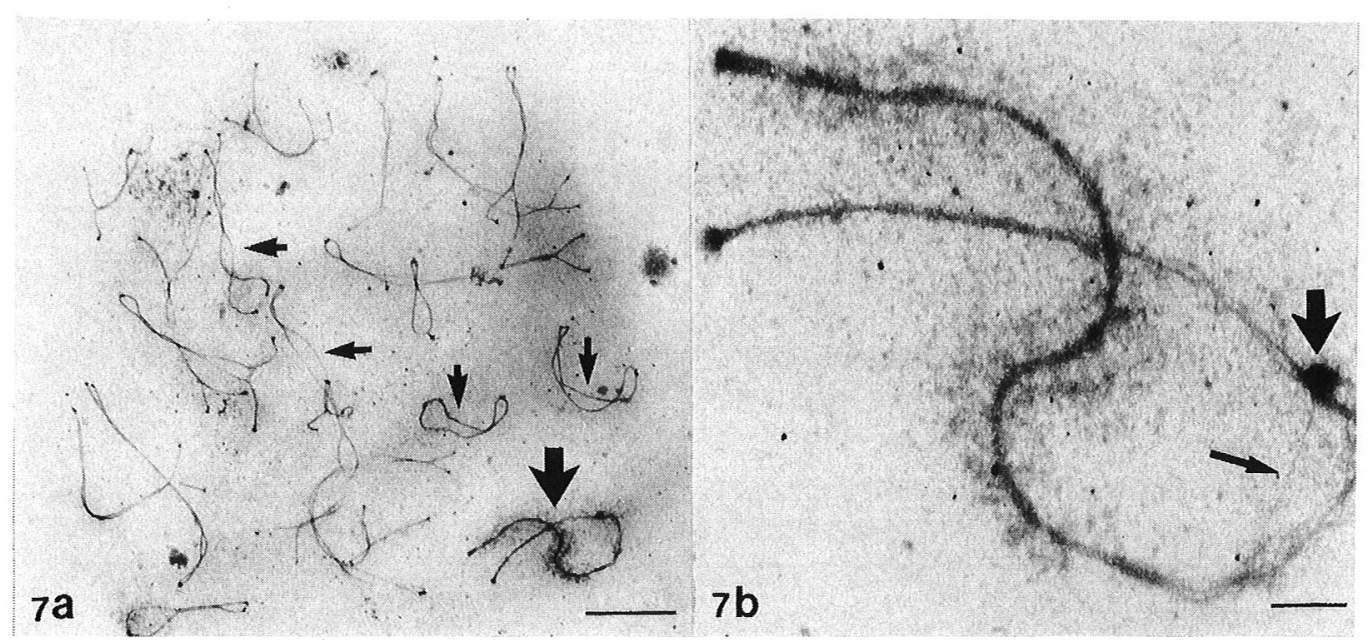

Fig. 7. Electron micrographs of synaptonemal complexes of Turkish hamster spermatocytes. (PTAstained.) a) Overview of nuclei showing diplotene stage; desynapsis of autosomal bivalents is progressing along the axes (small arrow). Large arrow indicates XY pair. b) Enlargements of XY pair showing type 6 configuration; the XY pairing shows end-to-end joining at 2 attachment plaques (large arrow) and a fine strand (small arrow) still can be identified.

Table 1. The original data of relationship between 6 meiotic stages/substages and 6 types of XY pairing conformation (No. of cells)

\begin{tabular}{lrrrrrrrrrrrrrrrr}
\hline Type & T1 & \multicolumn{1}{c}{ T2 } & \multicolumn{2}{c}{ T3 } & & T4 & & T5 & & T6 & & \multicolumn{2}{c}{ Sub-total } \\
\hline Stage/ & & & & & & & & & & & & & & \\
Substage & A & B & A & B & A & B & A & B & A & B & A & B & A & B \\
\hline P1 & 1 & 2 & & & & & & & & & & & 1 & 2 \\
P2 & 10 & 11 & 6 & 8 & 2 & 4 & & & & & & & 18 & 23 \\
P3 & 17 & 15 & 15 & 13 & 3 & 6 & & & & 1 & & & 35 & 35 \\
P4 & 6 & 15 & 7 & 4 & 9 & 3 & & & 2 & 9 & & & 24 & 31 \\
P5 & 3 & 2 & 2 & 1 & 2 & 1 & 2 & 3 & 7 & 5 & 5 & & 21 & 12 \\
D & 0 & 0 & 1 & 0 & & & & 4 & 6 & 4 & 6 & 4 & 13 & 12 \\
\hline Sub total & 37 & 45 & 31 & 26 & 16 & 14 & 2 & 7 & 15 & 19 & 11 & 4 & 112 & 115 \\
\hline
\end{tabular}

P1-P5, Substages of pachytene cells; D, Diplotene; T1-T6, Types of XY pairing conformation; A, lst spreading technique with PTA-staining method; B, 2nd spreading technique with silver-nitrate staining method.

Table 2. Incidence of cells where correlations existed between 4 meiotic stages/substages and 4 types of XY pairing conformation

\begin{tabular}{lrccrc}
\hline \multicolumn{1}{c}{ Type } & T1 & T2 + T3 & T4 + T5 & T6 & Total \\
Stage/Substage & & & & & \\
\hline Early pachytene (P1+P2) & 24 & 20 & 0 & 0 & 44 \\
Mid pachytene (P3) & 32 & 37 & 1 & 0 & 70 \\
Late pachytene (P4+P5) & 26 & 29 & 28 & 5 & 88 \\
Diplotene & 0 & 1 & 14 & 10 & 25 \\
\hline Total & 82 & 87 & 43 & 15 & 227 \\
\hline
\end{tabular}

P1-P5, Substages of pachytene cells; D, Diplotene cells; T1-T6, Types of XY pairing conformation. 


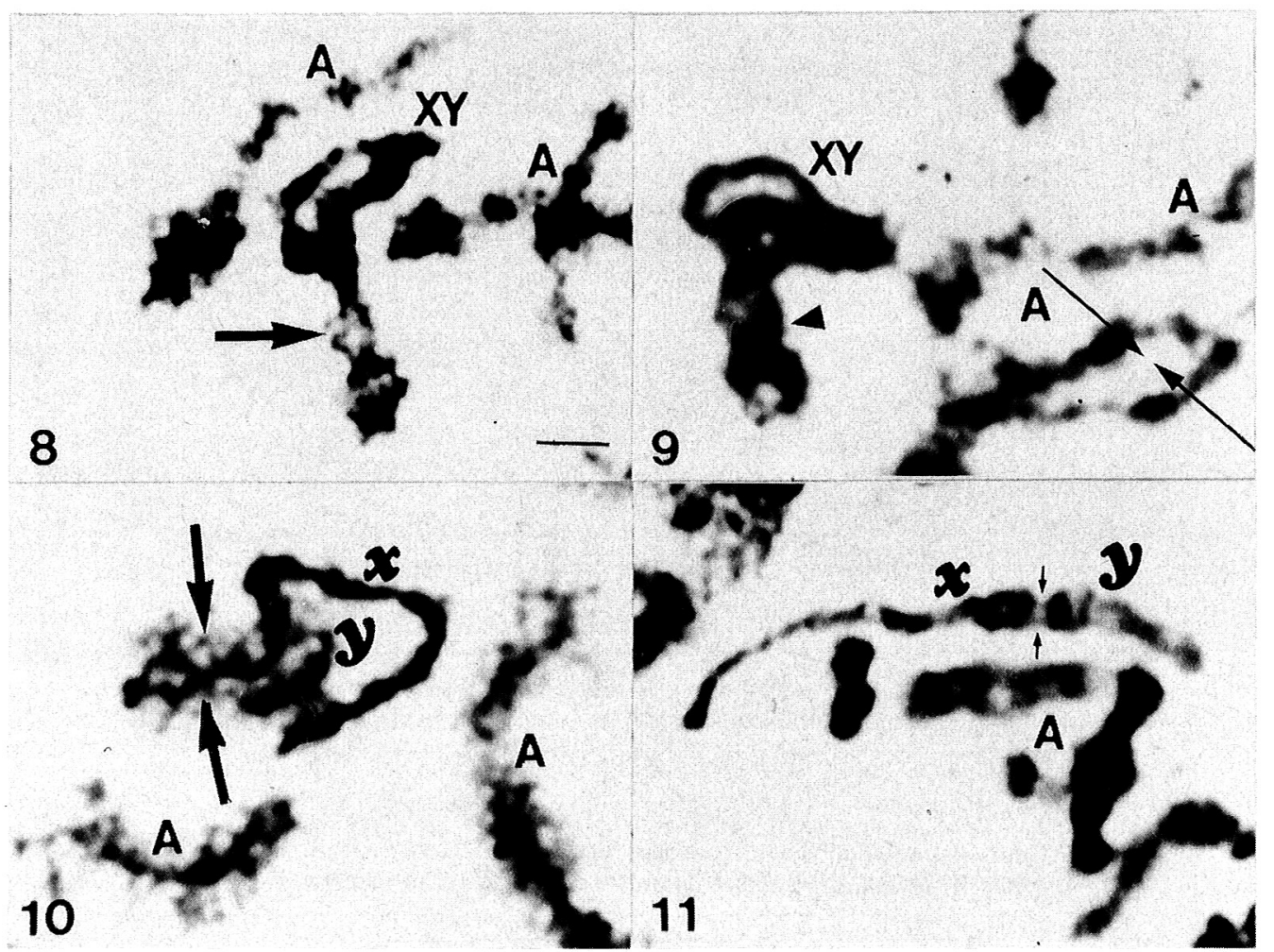

Fig. 8-11. Light micrographs of $X Y$ pairs in silver stained cytogenetic preparations of Turkish hamster spermatocytes. XY,XY pair; X,X-chromosome; X,Y chromosome; A, Autosomal bivalent. Scale represents $80 \mu \mathrm{m}$. In Fig. 8, the relaxation occurs along the XY paired segment (arrow). In Fig. 9, the cross connection of the $\mathrm{X}$ and $\mathrm{Y}$ axes is seen in the paired segment (arrow head). Initiation of disjunction occurs in some autosomal bivalents (arrows). Fig. 10 shows the disjunction and the cross connection of the $\mathrm{X}$ and $\mathrm{Y}$ axes are evident (arrows). In Fig. 11, the $\mathrm{X}$ and $\mathrm{Y}$ chromosomes are still connected with the fine threads (arrows) when they show an end-to-end association at the diakinesis stage.

had disappeared.

After the XY pairing conformations were classified as described above, six meiotic stages/substages of these nuclei were determined by autosomal criteria. The initial data of cell scores of the 6 types of the XY pairing conformations and 6 meiotic stages/substages of the nuclei are shown in Table 1. A nearly identical result was obtained from the 2 different spreading techniques. In order to compare further the correlations between the types of the XY pair and the stages/substages of the nuclei, the data obtained from 2 spreading methods were pooled and the substages of P1, P2 and P4, P5 were combined as early and late pachytene stages respectively, as Greenbaum $e t$ al. [16] suggested. Similarly the types of the XY pairs were also re-classified into 4 types by pooling $\mathrm{T} 2$, $\mathrm{T} 3$ and T4, T5 respectively so that the classification would be simplified and based only on the positions of the stranded connections regardless of the number of strands. The correlation between the 4 meiotic stages/substages and 4 types of the $\mathrm{XY}$ pairs are shown in Table 2. The side-by-side pairing conformation without any stranded connection (T1) was principally seen during early to mid pachytene stages. The side-by-side XY pair with stranded connections in the central space of the two axes around the border of the differential and undifferential segments (T2+T3) was also found at early pachytene $(\mathrm{P} 1+\mathrm{P} 2)$ and increased in number throughout mid (P3) to late (P4+P5) pachytene stages. The stranded connection observed near the attachment plaques $(\mathrm{T} 4+\mathrm{T} 5)$ was found primarily after late pachytene (P4+P5) and the end-to-end association of the XY pair (T6) appeared mainly at the diplotene stage (D). A similar findings were seen by light microscopic observations of the cytogenetic preparations (Figs. 8-11). The relaxation of the pairing segment of the XY bivalent progressed gradually through 
pachytene into diplotene (Figs. 8-10). Although there was a limit in the resolution of the light microscopy, two separated axes with a cross connecting structure were clearly discernible in the paired region of the XY bivalent. At the diakinesis stage, the $\mathrm{X}$ and $\mathrm{Y}$ chromosomes were still connected with 2 fine threads in the distal portions to the stage of end-to-end association (Fig. 11). Thus, the male Turkish hamster has provided a unique system for visualizing by electron and light microscopy the chiasma between the $\mathrm{X}$ and $\mathrm{Y}$ chromosomes throughout the development of pachytene and diplotene spermatocytes.

\section{Discussion}

The possibility of crossing over between the XY chromosomes has been generally accepted, yet structural proof of chiasma formation has largely depended on circumstantial evidence. With exceptions only in 3 hamster species; Chinese (Cricetulus griseus) [32-34]; European (Cricetulus cricetus) [33], and Armenian (Cricetulus migratorius) [35], previous light microscopic observations of air-dried preparations have demonstrated the $\mathrm{X}$ and $\mathrm{Y}$ chromosomes in most mammals in an end-to-end association without visible chiasma formation at the diakinesis and metaphase I stages. Although Ohno et al. [36] assumed that this end-to-end joining represented the result of terminalization of a near terminal chiasma, it was not possible to trace back the formation and the sequential changes of chiasma with the light microscope because structural details of the XY pair were obscured throughout most of pachytene stage [35]. Thus, it was not possible to distinguish between the previous existence of partial synapsis and complete lack of synapsis once the sex chromosomes appeared in an end-to-end association at diakinesis. On the other hand, Solari [37-39] using light and electron microscopy described the three-dimensional reconstruction of the mouse and the Armenian hamster XY pair and strongly suggested the existence of the chiasma in the XY pair as well as in homologous autosomes. The basis of this was the presence of a synaptonemal complex followed by the appearance of the fibrillar material which replaced the structure in the central space of the paired region in 2 axes. Although several studies $[37,40]$ stated that the presence of a synaptonemal complex was a prerequisite for the formation of a chiasma, it was not in itself sufficient for such formation as pointed out by Solari $[37,38]$. Thus, sequential electron microscopic observations of the development of the chiasma had not been available. By following the entire progression of the XY synaptonemal complex through pachytene to diplotene in the present study, $\pm 64 \%$ of the analyzed XY pairs through pachytene showed 1 or 2 strands connecting 2 axes on the central space of the pairing segments with all the spreading and the staining methods used.

Doubleness or multistranded structures in the lateral elements of the synaptonemal complex of spread preparations have been reported in several species including mouse [41-45], rat [41, 43] and Chinese hamster [46, 47]. Their biological role or genetic function has not been defined, but a possible role of such structures in crossing over was inferred. Moens [48] noted the possible relationship between the lateral element cross connections and the chiasmata in rat spermatocytes synaptonemal complex. Wahrman [45] suggested that such chromatid axes participated in genetic exchange rather than that the lateral elements were themselves involved in recombination. Heyting et al. [43] who observed isolated rat synaptonemal complexes, suggested that a local thickening of the lateral elements where 2 axes were still together at the diplotene stage might mark the sites where crossing over had occurred. In the present work, by observing the sequence from early pachytene to diplotene spermatocytes in the Turkish hamster, the fine fibrillar strands extending from 1 axis to another clearly showed a structural connection between the $\mathrm{X}$ and $\mathrm{Y}$ chromosomes. This finding was supported by the observations on the XY pairs of the cytogenetic preparations in which the sequential change from the relaxation of the pairing, the appearance of the cross connection, to the disjunction of the 2 axes toward an end-to-end relationship was seen from pachytene to diakinesis. The sequential appearance of the stranded structures and the movement of the positions where the connections occurred might suggest that a cross-over and a resultant chiasma had occurred between the sex chromosomes.

When chiasma formation was observed as early as mid-pachytene in the $\mathrm{XY}$ pairs, the autosomal bivalents were still tightly paired (Figs. 3a, 3b). 
Prior to the $\mathrm{XY}$ pair displaying end-to-end joining, the autosomes had begun to initiate their pairing relaxation with resultant desynapsis and chiasma appearance. This timing lag which existed between the sex and the autosomal chromosomes is paralleled in the similar precocious desynapsis of the XY pairs of mouse [37], human [49, 50], and Chinese hamster [47]. It was believed to be a barrier to the occurrence of more crossing over between the 2 axes [18]. Apparently such early disjunction of the XY pair also exists in Turkish hamster. Moreover, the present observations demonstrate that the precocious behavior of the XY pair was accompanied by the early formation and appearance of the chiasma.

Detection of the XY chiasma by synaptonemal complex analysis may also be an alternate possibility related to the long length of the XY bivalent. Solari [38] stated that the architecture of the sex chromosomes does not differ between species showing a chiasma and the species showing an end-to-end attachment. He reported [38] that a longer synaptonemal complex existed between the $\mathrm{X}$ and $\mathrm{Y}$ in the Armenian hamster than mouse and man. This species has been recognized as one of those possessing the most well-defined chiasma formation between the $\mathrm{XY}$ in mammals reported at that time [35, 38]. A distinct chiasma formation between the XY pair observed in the present study may simply be due to the existence of a longer synaptonemal complex as Solari [38] pointed out. However, the possibility of a more complex mechanism such as a gonosomal-autosomal rearrangement $[51,52]$ or an evolution from a pair of homologous $[15,53]$ involved in the XY pair of this species cannot be excluded.
Holm and Rasmussen [40] claimed that with respect to the problem of the terminalization of such an exchange in autosomes, chiasma remains at their original position until diakinesis whereafter terminalization may occur. This concept has not been confirmed in the XY pairs of most species. Even if the end-to-end joining of the XY pairs represented a terminalization of a near terminal chiasma as Ohno et al. [36] suspected, the position where the chiasma may have originally occurred, could not have been detected before diakinesis. In the present study of Turkish hamster, because a chiasma formation was observed as early as mid-pachytene, movement toward the chromatid end from the original position could be observed, providing possible evidence for terminalization.

However, the concept of chiasma terminalization [54-56] has been challenged by several works $[20,22,24,57]$. From the results of an analysis of chiasma behavior in spermatocytes of male mice, Imai \& Moriwaki (1982) strongly suggested that no terminalization occurs in those interstitial chiasmata. Furthermore, they assumed that the terminal association seen in several autosomal bivalents and $\mathrm{XY}$ pairs are achiasmatic. The latter assumption has been partially supported by their series of studies on $\mathrm{X}-\mathrm{Y}$ chromosome dissociation in intersubspecies hybrid mice [19, 21, 23]. Thus, whether the movement of crossing strands toward the terminal ends of XY pairs observed in the present study of Turkish hamster represented a chiasma terminalization or is simply related with the associated axes as desynapsis increases, remains uncertain.

This work was supported by NICHD. 


\section{References}

1. Koller PC, Darlington CD. The genetical and mechanical properties of the sex chromosomes. Rattus norvegicus. J Genet 1934; 29: 159-173.

2. Burgoyne PS. Genetic homology and crossing over in the $\mathrm{X}$ and $\mathrm{Y}$ chromosomes of mammals. Hum Genet 1982; 61: 85-90.

3. Burgoyne PS. Mammalian X and Y crossover. Nature 1986; 319: 258-259.

4. Burgoyne PS. The genetics of sex in development. In: Hamilton D, Naftolin F (eds.), Basic Reproductive Medicine, vol. 1: Basis and development of reproduction. Cambridge, London: MIT Press; $1981 ; 1-31$.

5. Singh L, Jones KW. Sex reversal in the mouse (Mus musculus) is caused by a recurrent nonreciprocal crossover involving the $\mathrm{X}$ and an aberrant $\mathrm{Y}$ chromosome. Cell 1982; 28: 205-216.

6. Cooke HJ, Brown WRA, Rappold A. Hypervariable telomeric sequences from the human sex chromosomes are pseudoautosomal. Nature 1985; 317: 687-692.

7. Goodfellow PJ, Darling SM, Thomas NS, Goodfellow PN. A pseudoautosomal gene in man. Science 1986; 234: 740-743.

8. Harbers K, Soriano P, Muller U, Jaenisch R. High frequency of unequal recombination in pseudoautosomal region shown by proviral insertion in transgenic mouse. Nature (Lond.) 1986; 324: $682-685$.

9. Keitges E, Rivest M, Siniscalco M, Gartler SM. $\mathrm{X}$-linkage of steroid sulphatase in the mouse is evidence for a functional Y-linked allele. Nature (Lond.) 1985; 315: 226-227.

10. Page DC, Mosher R, Simpson EM, Fisher EMC, Mardon G, Pollack J, McGillivry B, Dela Chapelle A, Brown LG. The sex-determining region of the human $\mathrm{Y}$ chromosome encodes a finger protein. Cell 1987; 51: 1091-1104.

11. Rouyer F, Simmler MC, Johnson C, Vergnaud G, Cooke HJ, Weissenbach J. A gradient of sex linkage in the pseudoautosomal region of the human sex chromosomes. Nature 1986; 319: 290-295.

12. Simmler MC, Rouyer F, Vergnaud G, NystromLahti M, Ngo KY, De la Chapelle A, Weissenbach J. Pseudoautosomal DNA sequences in the pairing region of the human sex chromosomes. Nature 1985; 37: 692-697.

13. Soriano PH, Keitges EA, Schoroderet DF, Harbers K, Gartler SM, Jaenisch R. High rate of recombination and double crossovers in the mouse pseudoautosomal region during male meiosis. Proc Natl Acad Sci 1987; 84: 7218-7220.

14. Weber B, Weissenbach J, Schempp W. X-Y cros- sing over in the chimpanzee. Hum Genet 1988; 80: 301-303.

15. Ellis N, Goodfellow PN. The mammalian pseudoautosomal region. Trends in Genet 1989; 5: 406410.

16. Goodfellow PN, Darling SM. Genetics of sex determination in man and mouse. Development 1988; 102: 251-258.

17. Weissenbach J, Levilliers J, Petit C, Rouyer F, Simmler MC. Normal and abnormal interchanges between the human $\mathrm{X}$ and $\mathrm{Y}$ chromosomes. Development 1987; 101 (suppl): 67-74.

18. Ashley T. A re-examination of the case for homology between the $\mathrm{X}$ and $\mathrm{Y}$ chromosomes of mouse and man. Hum Genet 1984; 67: 372-377.

19. Imai HT, Matsuda TS, Moriwaki K. High frequency of X-Y chromosome dissociation in primary spermatocytes of $F_{1}$ hybrids between Japanese wild mice (Mus musculus molossinus) and inbred laboratory mice. Cytogenet Cell Genet 1981; 29: $166-175$.

20. Imai HT, Moriwaki K. A re-examination of chiasma terminalization and chiasma frequency in male mice. Chromosoma (Berl.) 1982; 85: 439-452.

21. Imai HT, Wada MY, Moriwaki K. The sex chromosome association (Sxa) gene is located on the X-chromosome in mice. Jpn J Genet 1990; 65: 65-69.

22. Kanda $\mathbf{N}$, Kato $\mathbf{H}$. Analysis of crossing over in mouse meiotic cells by BrdU labelling technique. Chromosoma (Berl.) 1980; 78: 113-121.

23. Matsuda Y, Imai HT, Moriwaki K, Kondo $\mathbf{K}$, Bonhomme F. X-Y chromosome dissociation in wild derived Mus musculus subspecies, laboratory mice, and their $\mathrm{F}_{1}$ hybrids. Cytogenet Cell Genet 1982; 34: 241-252.

24. Maudlin I, Evans EP. Chiasma distribution in mouse oocytes during diakenesis. Chromosoma (Berl.) 1980; 80: 49-56.

25. Trés LL, Kierszenbaum AL. Meiotic chromosomes of mouse spermatocytes: Identification of bivalents, lampbrush organization and transcription activities. In: Jagiello GM and Vogel HJ(ed.), Bioregulators of Reproduction. New York: Academic Press; 1981: 229-256.

26. Sung WK, Jagiello GM. A technique for light and electron microscopy of the synaptonemal complex of the mouse oocyte. Can J Genet Cytol 1982; 24: 675-680.

27. Moses MJ. Synaptonemal complex karyotyping in spermatocytes of Chinese hamster (Cricetulus griseus). I. Morphology of the autosomal complement in spread preparations. Chromosoma (Berl.) 1977a; 60: 99-125. 
28. Fletcher JM. Light microscopy analysis of meiotic prophase chromosomes by silver staining. Chromosoma 1979; 72: 241-248.

29. Greenbaum IF, Hale DW, Fuxa KP. The mechanism of autosomal synapsis and the substaging of zygonema and pachynema from deer mouse spermatocytes. Chromosoma (Berl.) 1986; 93: 203-212.

30. Sung WK, Jagiello GM. Synaptonemal complex karyotype of the pachytene spermatocytes and oocytes of the Turkish hamster (Mesocricetus brandti) Caryologia 1992; 45: 325-337.

31. Solari AJ. Synaptonemal complexes and associated structures in microspread human spermatocytes. Chromosoma (Berl.) 1980; 81: 315-337.

32. Fraccaro M, Gustavsson I, Hultén J, Lindstein J, Tiepolo L. Late-replicating $\mathrm{Y}$ chromosome in spermatogonia of the Chinese hamster (Cricetulus griseus). Cytogenetics 1969; 8: 263-271.

33. Fredga K, Santesson B. Male meiosis in the Syrian, Chinese, and European hamsters. Hereditas 1964; 52: $36-48$.

34. Utakoji T. On the homology between the $\mathrm{X}$ and the $\mathrm{Y}$ chromosomes of the Chinese hamster. Chromosoma (Berl.) 1966; 18: 449-454.

35. Lavappa KS, Yerganian G. Spermatogonial and meiotic chromosomes of the Armenian hamster, cricetulus migratorius. Exp Cell Res 1970; 61: 159-172.

36. Ohno S, Kaplan W, Kinosita R. On the end-to-end association of the $\mathrm{X}$ and $\mathrm{Y}$ chromosomes of Mus musculus. Exp Cell Res 1959; 18: 282-290.

37. Solari AJ. The spatial relationship of the X and Y chromosomes during meiotic prophase in mouse spermatocytes. Chromosoma (Berl.) 1970; 29: 217-236.

38. Solari AJ. The relationship between chromosomes and axes in the chiasmatic XY pair of the Armenian hamster (Cricetulus migratorius). Chromosoma (Berl.) 1974a; 48: 89-106.

39. Solari AJ. The behavior of the XY pair in mammals. Int Rev Cytol 1974b; 38: 273-317.

40. Holm P, Rasmussen SW. Human meiosis VII. Chiasma formation in human spermatocytes. Carlsberg Res Commun 1983; 48: 415-456.

41. Del Mazo J, Gil-Alberdi L. Multistranded organization of the lateral elements of the synaptonemal complex in the rat and mouse. Cytogenet Cell Genet 1986; 4: 219-224.

42. Dietrich AJJ, De Boer P. A sequential analysis of the development of the synaptonemal complex in spermatocytes of the mouse by electron microscopy using hydroxyurea and agar filtration. Genetica 1983; 61: 119-129.

43. Heyting C, Dietrich AJJ, Egbert JW, Redeker EJW, Vink ACG. Structure and composition of synaptonemal complexes, isolated from rat spermatocytes. Europ J Cell Biol 1985; 36: 307-314.

44. Trés LL. Extensive pairing of the XY bivalent in mouse spermatocytes as visualized by whole-mount electron microscopy. J Cell Sci 1977; 25: 1-15.

45. Wahrman J. Synaptonemal complexes-origin and fate. Chromosomes Today 1981; 7: 105-113.

46. Dresser MI, Moses MJ. Synaptonemal complex karyotyping in spermatocytes of the Chinese hamster (Cricetulus griseus) IV. Light and electron microscopy of synapsis and nucleolar development by silver staining. Chromosoma 1980; 76: 1-22.

47. Moses MJ. Synaptonemal complex karyotyping in spermatocytes of Chinese hamster (Cricetulus griseus). II. Morphology of the XY pair in spread preparations. Chromosoma (Berl.) 1977b; 60: 127137.

48. Moens PB. Laternal element cross connections of the synaptonemal complex and their relationship to chiasmata in rat spermatocytes. Can J Genet Cytol 1978; 20: 567-579.

49. Moses MJ, Counce SJ, Paulson DF. Synaptonemal complexes complement of man in spreads of spermatocytes, with details of the sex chromosome pair. Science 1975; 187: 363-365.

50. Solari AJ, Trés LL. The three-dimensional reconstruction of the XY pair of human spermatocytes. $J$ Cell Biol 1970; 45: 43-53.

51. Ortells MO, Reig OA, Brum-Zorilla N, Scaglia OA. Cytogenetics and karyosystematics of phyllotine rodents (Cricetidae, Sigmodontinae). I. Chromosome multiformity and gonosomal-autosomal translocation in Reithrodon. Genetica 1988; 77: 53-63.

52. Ratomponirina C, Viegas-Pequignot E, Dutrillaux B, Petter F, Rumpler Y. Synaptonemal complexes in Gerbillidae: probable role of intercalated heterochromatin in gonosome-autosome translocations. Cytogenet Cell Genet 1986; 43: 161-167.

53. Matthey R. Les bases cytologiques de l'heredite «relativement» liee au sexe chez les mammiferes. Experientia XIII: 341-380.

54. Janssen FA. Spermatogenese dans les Batraciens. V. La théorie de la chiasmatype. Nouvelle interprétation des cinéses de maturation. Cellule 1909; 25: 387-411.

55. Janssen FA. La chiasmatypie dans les insectes. Cellule 1924; 34: 135-359.

56. Darlington CD. Recent advances in cytology. Blakiston's Philadelphia 1932.

57. Imai HT, Crozier RH. Quantitative analysis of directionality in mammalian karyotype evolution. Amer Nat 1980; 116: 537-569. 